\title{
Emerging treatment options for short bowel syndrome: potential role of teduglutide
}

This article was published in the following Dove Press journal:

Clinical and Experimental Gastroenterology

18 August 2011

Number of times this article has been viewed

\author{
Cheng $T$ Tee ${ }^{1,2}$ \\ Katharina Wallis ${ }^{1,3}$ \\ Simon M Gabe ${ }^{1,4}$ \\ 'Lennard-Jones Intestinal Failure Unit, \\ St Mark's Hospital and Academic \\ Institute, Harrow, UK; ${ }^{2}$ Antigen \\ Presentation Research Group, \\ Imperial College London, Northwick \\ Park and St Mark's Campus, Harrow, \\ UK; ${ }^{3}$ West Hertfordshire Hospitals \\ NHS Trust, Watford, UK; ${ }^{4}$ Division \\ of Surgery, Oncology, Reproductive \\ Biology and Anaesthetics, Imperial \\ College Healthcare, London, UK
}

Correspondence: Cheng T Tee Antigen Presentation Research Group, Level 7, St Mark's Hospital and Academic Institute, Watford Road,

Harrow HAI 3UJ, UK

Tel +440208869365।

Fax +440208 8693532

Email c.tee@imperial.ac.uk
Introduction: Current medical management of short bowel syndrome (SBS) involves the use of lifelong parenteral nutrition (PN). Glucagon-like peptide-2 (GLP-2), an important intestinotrophic growth factor has been shown to increase intestinal absorption in SBS through augmentation of post-resection intestinal adaptation. This may lead to the reduction of PN dependence in patients with SBS.

Areas covered in review: Advancing research of GLP-2 physiology has spurred the growing understanding of the diverse effects of GLP-2. The development of the degradation resistant GLP-2 analog, teduglutide (Gattex ${ }^{\mathrm{TM}}$, NPS Pharmaceuticals, Bedminster, NJ), has allowed its exploration as a therapeutic agent in a variety of clinical settings. Recent multicenter, placebocontrolled studies of GLP-2 in SBS patients demonstrate meaningful reductions in PN requirements with good safety profiles. The reparative and immunomodulatory effects of teduglutide may also be beneficial in patients with inflammatory bowel disease (IBD). Safety concerns about possible carcinogenic properties during long-term use require ongoing evaluation.

Summary: GLP-2 appears to offer a novel adjuvant treatment modality for SBS. Promise for its use in other clinical settings like IBD has been shown in small pilot studies.

Keywords: glucagon-like peptide-2, intestinal failure, intestinal adaptation, parenteral nutrition

\section{Introduction}

Short bowel syndrome (SBS) is defined by a combination of symptoms and signs that occur after extensive surgical resection of the intestine. This highly disabling condition is characterized by malabsorption of both fluid and nutrients and, left untreated, can lead to dehydration, malnutrition, and weight loss. The term intestinal failure (IF) applies when an adequate balance of nutrients and water cannot be maintained without dietary support. IF often remains a short-term problem in the postoperative period. However, a small number of patients will require long-term parenteral nutrition (PN) or, in selected cases, intestinal transplantation. Such patients will typically have less than $100 \mathrm{~cm}$ of small bowel leading to an end-stoma or less than $50 \mathrm{~cm}$ connected to a functioning colon. Although PN has revolutionized IF treatment, it has a significant impact on quality of life and carries considerable risks, mainly hepatic failure, central vein thrombosis, and recurrent sepsis, all of which will reduce life expectancy. Survival following intestinal transplantation is still inferior to that of long-term PN due to the high incidence of graft rejection and other postoperative complications. ${ }^{1}$

Soon after surgical resection with resulting loss of surface area, the intestine physiologically attempts to increase absorption to maintain homeostasis. This process 
of intestinal adaptation occurs through structural (villous cell hyperplasia, increased crypt depth, and intestinal dilatation) and functional (increased mucosal enzyme activity and reduction of intestinal transit) mechanisms leading to a gradual increase in absorptive capacity. This period of adaptation is thought to last up to 24 months. Nutritional (eg, glutamine) and non-nutritional (eg, growth factors) substances have been implicated in promoting this adaptive response. In the last decade, most IF research has been focused on exploring the potential of these substances as supportive IF treatment. However, clinical trials so far have not demonstrated reproducible or meaningful clinical benefits with the use of glutamine or growth hormone. ${ }^{2-5}$

Current supportive medical management includes the use of agents that reduce secretion (H2 receptor blockers, proton pump inhibitors, and octreotide) and motility (codeine, opium, lomotil, and loperamide). ${ }^{6}$ The goal is to reduce the total stool output to $<2 \mathrm{~L}$ per day. Dietary advice to maximize intestinal absorption is highly beneficial but needs to be tailored to the anatomy of the residual bowel.
The naturally occurring gut hormone, glucagon-like peptide-2 (GLP-2), is a pleiotropic intestinotrophic hormone that enhances digestive and absorptive capacity. ${ }^{7}$ Recent advances in our understanding of the basic science of GLP-2 have led to its exploration as a potential 'first in class' therapeutic drug for SBS.

\section{Glucagon-like peptide-2 and teduglutide}

Glucagon-like peptide-2 is a 33-amino acid peptide with an estimated molecular mass of $3765.8 .^{8}$ GLP-2 is derived from the post-translational processing of pro-glucagon, a large prohormone that is mainly expressed in the pancreas, intestine, and brain (Figure 1). In the intestine, biologically active GLP-2 $2^{1-33}$ is secreted from enteroendocrine L-cells of the ileum and colon in response to nutritional, hormonal, and neural stimulation. Human and animal studies have revealed that dietary fiber and short-chain fatty acids, carbohydrates, and fats are potent stimulators of GLP-2 secretion. ${ }^{9}$

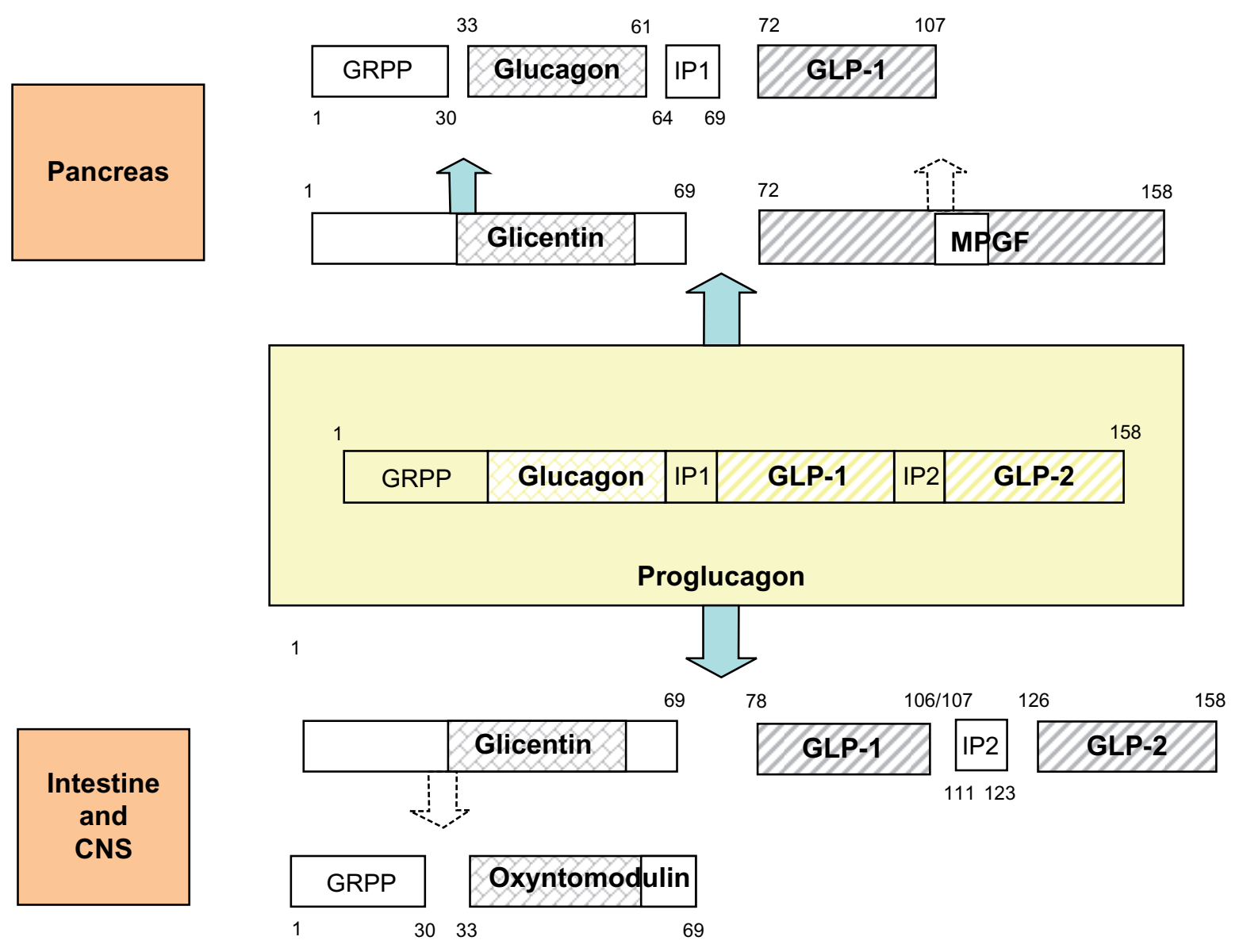

Figure I Proglucagon contains three homologous hormonal sequences, glucagon/GLP-I/GLP-2, and is separated by intervening peptides IPI/IP2. Proglucagon is processed differentially in pancreas, intestine, and CNS.

Abbreviations: CNS, central nervous system; GLP, glucagon-like peptide; GRPP, glicentin-related polypeptide; IP, intervening peptide; MPGF, major proglucagon fragment. 
GLP-2 exerts a wide variety of effects on the gastrointestinal tract and is a key mediator of intestinal adaptation. ${ }^{10}$ In animal studies, GLP-2 treatment induces mucosal growth in the small and large intestine through an increase in crypt cell proliferation and a reduction of villous cell apoptosis (Figure 2). ${ }^{11,12}$ This increase in mucosal mass is accompanied by enhanced functional absorptive capacity. ${ }^{13}$ Other GLP-2mediated effects include inhibition of gastric emptying and acid secretion, ${ }^{14,15}$ reduction of intestinal permeability, ${ }^{16,17}$ anti-inflammatory actions, ${ }^{18}$ and stimulation of mesenteric blood flow. ${ }^{19}$

Specific G-protein coupled GLP-2 receptors (GLP-2Rs) are found in abundance in the proximal small intestine and have been demonstrated on enteroendocrine cells, ${ }^{20}$ enteric neurons, ${ }^{19}$ and subepithelial myofibroblasts. ${ }^{21}$ The apparent lack of GLP-2Rs on the proliferative crypt cells suggests that the effects of GLP-2 are mediated through a variety of downstream effectors (Figure 3). ${ }^{22}$ Insulin-like growth factor-1 appears to be essential for GLP-2-induced intestinal epithelial proliferation, ${ }^{23}$ and nitric oxide might be a key mediator in GLP-2-induced upregulation of intestinal blood flow. ${ }^{19}$ Vascular endothelial growth factor and transforming growth factor- $\beta$ have been linked to GLP-2-induced wound repair. ${ }^{24}$ The release of vasoactive intestinal peptide (VIP) from enteric neurons appears to mediate some of the anti-inflammatory effects of GLP-2. ${ }^{25}$ For further, in-depth discussion into the complex interplay of GLP-2 and its downstream mediators the authors of this paper recommend a recent review by Rowland and Brubaker. ${ }^{26}$

GLP-2 has a short half-life of approximately 7 minutes in humans and undergoes N-terminal truncation by the proteolytic enzyme dipeptidyl peptidase IV (DPPIV) to the
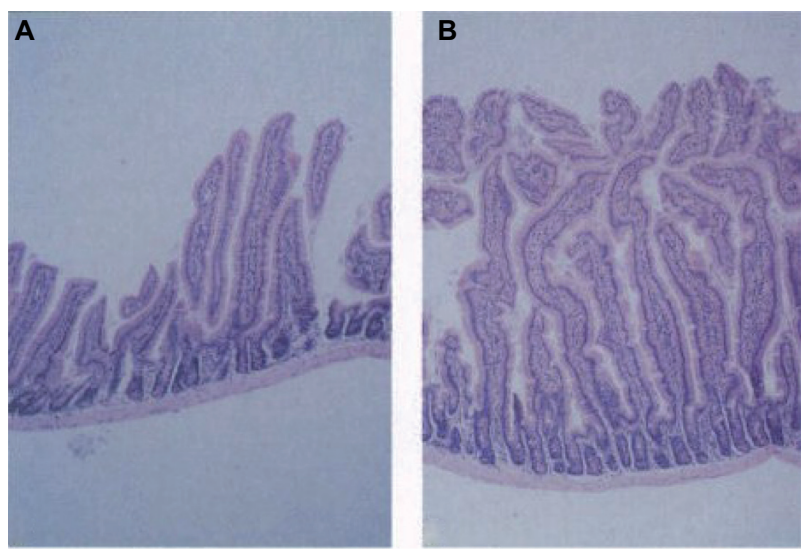

Figure 2 Effect of GLP-2 on murine small intestine. Histological appearance of small intestine epithelium from control (A) and GLP-2-injected (10 days) (B) mice. Reproduced with permission from Drucker et al."

(C) Copyright 1996 National Academy of Sciences, USA.

Abbreviation: GLP, glucagon-like peptide.

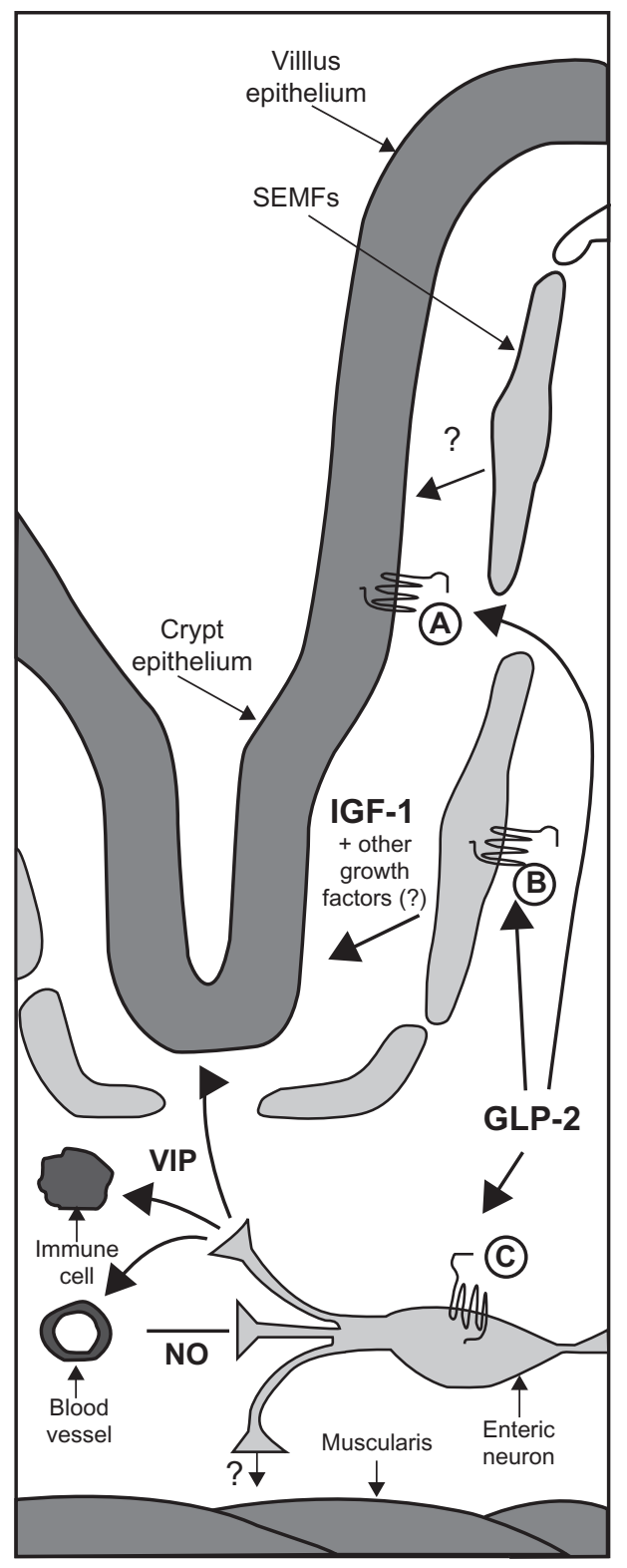

Figure 3 Proposed model for the indirect mechanisms of GLP-2 action in the intestine. Expression of the GLP-2R in intestinal endocrine cells (A), intestinal SEMFs (B), and enteric neurons (C) suggests that GLP-2 acts indirectly to produce its diverse actions in the intestine. IGF-I is critical for the ability of GLP-2 to induce intestinal growth and activate crypt cell proliferation. GLP-2-mediated enteric neuronal signaling enhances intestinal blood flow through a mechanism involving NO production and has anti-inflammatory actions through VIP.

Used with permission from Dubé et al, American Journal of Physiology-Endocrinology and Metabolism, Vol 293; E460-E465, 2007.22

Abbreviations: GLP-2, glucagon-like peptide-2; GLP-2R, glucagon-like peptide-2 receptor; IGF, insulin-like growth factor; NO, nitric oxide; SEMF, subepithelial myofibroblast; VIP, vasoactive intestinal polypeptide.

biologically inactive form GLP-2. ${ }^{3-33}$ Blocking of DPPIV degradation, either through glycine substitution in position 2 , as in teduglutide, or through adjuvant use of DPPIV inhibitors extends the use of GLP-2 half-life and confers greater biological potency. DPPIV inhibition enhances the intestinotrophic effect of GLP-2 in rats and mice. ${ }^{28}$ 
Teduglutide [Gly] GLP-2 (Gattex ${ }^{\mathrm{TM}}$, NPS Pharmaceuticals, Bedminster, NJ), a DPPIV-resistant analog of GLP-2 lacking the N-terminal DPPIV cleavage site, has received orphan drug designation for the treatment of SBS from the United States Food and Drug Administration (FDA) and the European Medicines Agency. In 2007, NPS Pharmaceuticals granted Nycomed the rights to teduglutide outside of North America.

\section{Clinical trials of glucagon-like peptide- 2 in SBS}

The production of GLP-2 occurs in both the small and large bowel via the proglucagon-expressing enteroendocrine L-cells but is most abundant in the distal ileum. This may explain why functional adaptation appears more effective when the residual intestine is ileum rather than jejunum. Those with end jejunostomy and no colon, who have the poorest intestinal adaptation, have a markedly impaired postprandial GLP-2 response, presumably caused by a lack of functioning L-cells. ${ }^{29}$ In contrast, SBS patients with preserved colon exhibit increased fasting GLP-2 levels with a postprandial profile comparable to that of healthy controls. ${ }^{30}$ These findings, combined with strong evidence of a correlation between GLP-2 and intestinal adaptation in animal models, ${ }^{13}$ have raised hopes that GLP-2 therapy might be able to produce clinically meaningful enhancement of intestinal mass and function in patients with SBS.

Results of clinical trials so far have been encouraging. In initial uncontrolled open label studies of SBS patients, native GLP-2 and the long-acting analog teduglutide significantly improved intestinal wet-weight absorption by up to $1 \mathrm{~L}$ per day. This occurred in patients with end jejunostomy as well as in patients with a preserved colon, despite the latter group having near normal endogenous GLP-2 levels. ${ }^{31,32}$ The effects have been shown to be maintained for up to 2 years of treatment but will quickly wear off following treatment termination. The effect on energy absorption appears to be minor.

A recently published large multinational, randomized, placebo-controlled clinical trial of teduglutide in 83 post-resection SBS patients requiring $\mathrm{PN}$ at least three times per week for a minimum of 12 months, has also reported a positive outcome. Following a maximum 8-week optimization of PN with a 4-8-week stabilization period, patients were randomized into a 24-week treatment protocol with placebo $(\mathrm{n}=16), 0.05 \mathrm{mg} / \mathrm{kg} /$ day teduglutide $(\mathrm{n}=35)$ or $0.10 \mathrm{mg} / \mathrm{kg} /$ day teduglutide $(\mathrm{n}=32)$. The main measure of treatment response was a reduction in parenteral nutrition requirements from baseline of more than $20 \%$. The PN reductions were made in standardized fashion when the urine volume increased to a certain threshold. The increased urine production was considered as a surrogate marker for increased intestinal wet-weight absorption. The reported primary efficacy endpoint of the study, was based on a graded response score (GRS) that accounted for both intensity (reduction from baseline of $20 \%-100 \%$ ) and duration of response (response at weeks 16, 20, and 24). Although no statistical significance could be shown for the $0.10 \mathrm{mg} / \mathrm{kg} /$ day dose group, the GRS in the lower $0.05 \mathrm{mg} / \mathrm{kg} /$ day dose group were significantly better than in the placebo group $(P=0.007)$. Also, a $>20 \%$ reduction of $\mathrm{PN}$ was achieved in significantly more patients treated with the $0.05 \mathrm{mg} / \mathrm{kg} / \mathrm{day}$ teduglutide compared with placebo $(46 \%(16 / 35)$ vs $6 \%$ $(1 / 16), P=0.005) .{ }^{33}$ The difference between $0.10 \mathrm{mg} / \mathrm{kg} /$ day teduglutide and placebo was not significant ( $25 \%$ vs $6.3 \%$ responders: $P=0.161)$. Three patients were weaned off PN completely (two in the low- and one in the high-dose treatment group).

The apparent lack of significant treatment response using $0.10 \mathrm{mg} / \mathrm{kg} /$ day teduglutide is thought to be related to protocol restrictions that could not take into account decreased oral fluid intake, which occurred significantly more often in the high dose patient group, as well as a trend towards higher baseline PN requirements and shorter remnant short bowel. Post hoc evaluation of the net effect of teduglutide estimates a similar efficacy of both treatment doses resulting in increased liquid absorption of approximately $700 \mathrm{~mL}$ (4.9 L per week), which is very much in keeping with the results of the openlabel trials. The investigators also argue that the therapeutic benefit of teduglutide might have been underestimated by considering PN reduction as isolated endpoints, rather than taking into account additional positive effects on hydration and renal function. Teduglutide led to a significant increase in urine excretion in the low-dose group, and despite a reduced oral intake, urine output was maintained in the high-dose group. Although energy absorption was not quantified in this study, there was a small increase in body weight and lean body mass in both treatment groups, despite reduction in PN calorie provision. ${ }^{34}$ Plasma citrulline, a sensitive marker for enterocyte mass and function, increased with low- and highdose treatment but not with placebo, indicating a teduglutiderelated intestinotrophic effect. Accordingly, increased villous height was documented in both treatment groups.

The aforementioned study was followed up with a 28-week extension study that enrolled 65 patients that had completed the initial trial. The aim was to investigate 
whether the PN reductions could be maintained for up to 1 year, as well as evaluate drug safety and tolerability. The results have been published in abstract form, and the final publication is anticipated. ${ }^{35}$ Patients previously on active treatment were maintained on their dose, and the placebotreated patients were randomized to teduglutide 0.05 or $0.10 \mathrm{mg} / \mathrm{kg} /$ day. In the low-dose group, 12/16 (75\%) of initial responders maintained the $\mathrm{PN}$ reduction; ten of these had further reductions. In the high-dose group, 6/8 (75\%) of initial responders maintained the reduction, and two of these had a further reduction. Among the initially placebotreated subjects, 6/6 (100\%) responded to low-dose and 2/7 (28\%) to high-dose teduglutide. Mean absolute (relative) PN reductions from weekly baseline volume were $4.5 \mathrm{~L}(51 \%$, low-dose group) and $3.0 \mathrm{~L}$ (24\%, high-dose group). Four actively treated subjects were weaned off parenteral nutrition over the 1-year trial period.

Prior to application for FDA licensing of teduglutide as a first-in-class treatment for SBS, a further confirmatory Phase 3 study (STEPS - Study of Teduglutide in PNdependent Short-bowel syndrome) has just been completed. This study randomized $86 \mathrm{SBS}$ patients to either $0.05 \mathrm{mg} / \mathrm{kg}$ of teduglutide Gattex or placebo over a 24 -week period. The following unpublished data has been extracted from a recent NPS Pharmaceuticals press release (January 31, 2011). ${ }^{36}$ The primary efficacy endpoint was defined as the percentage of patients who achieved a $20 \%$ or greater reduction in weekly PN volume at weeks 20 and 24. In an intentionto-treat analysis, $63 \%$ (27/43) of teduglutide-treated patients responded compared with $30 \%(13 / 43)$ of placebo-treated patients $(P=0.002)$. Absolute weekly reduction of $\mathrm{PN}$ volume was also greater in the teduglutide group compared with placebo; $4.4 \mathrm{~L}$ (baseline of $12.9 \mathrm{~L}$ ) vs $2.3 \mathrm{~L}$ (baseline of $13.2 \mathrm{~L}$ ). Teduglutide was well tolerated with only four patients discontinuing the study due to adverse events (one teduglutide treated and three placebo treated). The adverse events were consistent with the pharmacological effects of the drug. More than $97 \%$ of the eligible participants opted to participate in the STEPS 2 open-label continuation study where all participants will receive an additional 24 months of teduglutide therapy.

These clinical studies indicate that teduglutide treatment can achieve clinically useful reductions of $\mathrm{PN}$ requirements in SBS patients, considered being stable on optimal conventional management.

Metabolic bone disease is highly prevalent in SBS patients on long-term PN. A mediating role for GLP-2 in the postprandial decrease in bone resorption has been suggested because GLP-2 injection reduces bone resorption marker s-CTX in healthy postmenopausal women. ${ }^{37}$ In a small group of SBS patients with jejunostomy, 35-day GLP-2 treatment decreased bone resorption markers and increased bone mass and bone mineral density (BMD). ${ }^{38}$ However, other studies were unable to replicate the beneficial effect of GLP-2 on bone BMD in SBS patients. ${ }^{39,40}$ The clinical role of GLP-2 in the treatment of osteoporosis therefore remains uncertain.

Crohn's disease is a leading cause for adult SBS. Accumulating evidence that GLP-2 has anti-inflammatory and reparative properties promoted the expansion of GLP-2 research into inflammatory bowel disease (IBD). Bioactive GLP-2 circulating concentrations are elevated in patients with active IBD compared with healthy controls, indicating an innate adaptive mechanism to the associated IBD intestinal damage.$^{41}$ A pilot, proof of concept study was carried out on patients with moderate to severely active Crohn's disease. This 8-week, randomized, placebo-controlled, dose-ranging trial, which recruited 100 patients, suggests a dose-dependent benefit in the reduction of the Crohn's Disease Activity Index (CDAI) score. In the highest dose group, $55.6 \%$ of patients went into remission vs $33.3 \%$ of the placebo group. No statistical significance was observed, however, and further research must determine whether these results relate to symptomatic disease control (by decreasing diarrhea) or reflect decrease in inflammatory activity and improved mucosal healing. The authors acknowledged the pilot nature of the study as well as the use of CDAI as the primary outcome. ${ }^{42}$ Further clinical studies with greater statistical power and more effective primary endpoints and longer trial period are much needed. Nevertheless, this trial provided promising data for the use of teduglutide as a novel treatment of intestinal diseases associated with compromised repair of the intestinal mucosa.

There has been a number of studies looking at rodent models of intestinal injury to explore the mechanisms of anti-inflammatory actions of GLP-2. ${ }^{18,25,44}$ One such study carried out by Sigalet et al, investigated the effects of GLP-2 treatment on rats with trinitrobenzene sulfonic acid-induced ileitis or dextran sulfate sodium-induced colitis. ${ }^{25}$ Apart from trophic effects of GLP-2, treatment also resulted in significant reduction in inflammatory markers, interleukin (IL)-1 $\beta$, interferon- $\gamma$, and tumor necrosis factor- $\alpha$, along with reduction in neutrophil activity. The use of a VIP antagonist confirmed that VIP appeared to mediate the anti-inflammatory properties of GLP-2. Subsequently, Ivory et al confirmed the anti-inflammatory actions of GLP-2 via use of IL-10 knockout $\left(I L-10^{-/-}\right)$ 
mouse model. This study showed that GLP-2 altered the mucosal response of inflamed intestinal epithelial cells and macrophages by activation of the suppressor of cytokine signaling-3 pathway, which antagonizes the IL-6-mediated increase in signal transducers and activators of transcription 3 signaling. The study also confirmed that this immunomodulatory effect was IL-10 independent. ${ }^{18}$ Translation studies "from bench to bedside" may allow the clinical use of teduglutide as both a trophic and an immunomodulatory/ immunosuppressive therapy in patients with SBS, in particular those who develop SBS from complications of Crohn's disease.

\section{Safety consideration}

Clinical studies so far report good drug compliance, tolerance, and safety of native GLP-2 and teduglutide, with no significant increase of serious adverse events. Most commonly reported adverse events are abdominal pain, bloating, constipation, nausea, injection site erythema, lower limb edema, stoma swelling, headache, and nasopharyngitis. Caution is suggested in patients with a history of abdominal pain and recurrent subacute obstruction. In cardiac failure, increased fluid absorption might lead to decompensation and fluid overload.

The therapeutic use of GLP-2 may require lifelong administration. Because of concerns that permanent stimulation of epithelial proliferation might initiate or accelerate malignant growth, research into the role of GLP-2 in carcinogenesis is ongoing. Low level GLP-2R expression has been demonstrated in cancer cell lines and human tumors including colonic adenocarcinomas. ${ }^{43,45}$ GLP-2 also exerts a direct proliferative effect on transformed cell lines despite apparent lack of a specific receptor. ${ }^{46}$ Recent in vivo studies in mouse cancer models have added to concerns that GLP-2 may play a role in both tumor initiation and progression. Mice that were pretreated with carcinogen azoxymethane showed an increase in number and size of aberrant crypt foci, presumed precursors of tumor development, when they were subsequently treated with GLP-2 vs controls treated either with saline or the GLP-2R antagonist GLP-2. ${ }^{3-33}$ Loss of mucin production in aberrant crypts (considered an important marker of dysplasia) and formation of adenocarcinomas were almost exclusively observed in GLP-2-treated mice but not in controls. ${ }^{47}$ In another model, GLP-2 injections increased the number and size of colonic adenomas in mice pretreated with the carcinogen 1,2-dimethylhydralazine. ${ }^{48}$ It has been argued that the effects of GLP-2 are present at the early stage of carcinogenesis, with no effect when adenomas and microadenomas have already developed, as in the APC min/+ mice model..$^{45}$ The precise implications of such studies for the development of colon cancer in humans remain incompletely understood. Until a clear safety profile for the long-term use of GLP-2 is established, a high level of vigilance is required. This should include mandatory screening of prospective patients for pre-malignant lesions (particularly colonic adenomas).

\section{Conclusion}

The ability of GLP-2 to enhance intestinal adaptation has been demonstrated in numerous animal models. There is now mounting clinical evidence that it can provide a new therapeutic strategy in SBS patients in whom it may pave the path towards reducing PN dependence. This, in turn, may lead to a reduction in PN-related complications as well as improving patients quality of life. GLP-2 appears safe and well tolerated in the short and medium term. Optimal indications, timing, and dose as well as possible gains from coadministration with other growth factors will need to be studied further, and long-term colonic surveillance studies must be considered to abate safety concerns.

Promising results of the ability of GLP-2 to restore mucosal integrity and possible immunomodulatory effects bade the question of its use in IBD.

\section{Acknowledgment}

The St Mark's Research Foundation has supported this work.

\section{Disclosure}

Dr Tee, Dr Wallis, and Dr Gabe have been investigators in the multinational trial of teduglutide in the management of SBS, sponsored by NPS Pharmaceuticals.

\section{References}

1. Pironi L, Forbes A, Joly F, et al. Survival of patients identified as candidates for intestinal transplantation: a 3-year prospective follow-up. Gastroenterology. 2008;135(1):61-71.

2. Scolapio JS, McGreevy K, Tennyson GS, Burnett OL. Effect of glutamine in short-bowel syndrome. Clin Nutr. 2001;20(4):319-323.

3. Scolapio JS, Camilleri M, Fleming CR, et al. Effect of growth hormone, glutamine, and diet on adaptation in short-bowel syndrome: a randomized, controlled study. Gastroenterology. 1997;113(4):1074-1081.

4. Seguy D, Vahedi K, Kapel N, Souberbielle J-C, Messing B. Low-dose growth hormone in adult home parenteral nutrition-dependent short bowel syndrome patients: a positive study. Gastroenterology. 2003; 124(2):293-302.

5. Szkudlarek J, Jeppesen PB, Mortensen PB. Effect of high dose growth hormone with glutamine and no change in diet on intestinal absorption in short bowel patients: a randomised, double blind, crossover, placebo controlled study. Gut. 2000;47(2):199-205. 
6. Nightingale J, Woodward JM; and Small Bowel and Nutrition Committee of the British Society of Gastroenterology. Guidelines for management of patients with a short bowel. Gut. 2006;55(Suppl 4):iv1-iv12.

7. Hornby PJ, Moore BA. The therapeutic potential of targeting the glucagon-like peptide-2 receptor in gastrointestinal disease. Expert Opin Ther Targets. 2011;15(5):637-646.

8. Hartmann B, Johnsen AH, Orskov C, Adelhorst K, Thim L, Holst JJ. Structure, measurement, and secretion of human glucagon-like peptide-2. Peptides. 2000;21(1):73-80.

9. Brubaker PL, Anini Y. Direct and indirect mechanisms regulating secretion of glucagon-like peptide-1 and glucagon-like peptide-2. Can J Physiol Pharmacol. 2003;81(11):1005-1012.

10. Scott RB, Kirk D, MacNaughton WK, Meddings JB. GLP-2 augments the adaptive response to massive intestinal resection in rat. Am JPhysiol. 1998;275:911-921.

11. Drucker DJ, Erlich P, Asa SL, Brubaker PL. Induction of intestinal epithelial proliferation by glucagon-like peptide 2. Proc Natl Acad $\mathrm{Sci}$ US A. 1996;93(15):7911-7926.

12. Burrin DG, Stoll B, Guan X, Cui L, Chang X, Holst JJ. Glucagonlike peptide 2 dose-dependently activates intestinal cell survival and proliferation in neonatal piglets. Endocrinology. 2005;146(1): 22-32.

13. Koopmann MC, Nelson DW, Murali SG, et al. Exogenous glucagonlike peptide-2 (GLP-2) augments GLP-2 receptor mRNA and maintains proglucagon mRNA levels in resected rats. JPEN J Parenter Enteral Nutr. 2008;32(3):254-265.

14. Meier JJ, Nauck MA, Pott A, et al. Glucagon-like peptide 2 stimulates glucagon secretion, enhances lipid absorption, and inhibits gastric acid secretion in humans. Gastroenterology. 2006;130(1):44-54

15. Nagell CF, Wettergren A, Pedersen JF, Mortensen D, Holst JJ. Glucagon-like peptide-2 inhibits antral emptying in man, but is not as potent as glucagon-like peptide-1. Scand J Gastroenterol. 2004;39(4): 353-358.

16. Benjamin MA, McKay DM, Yang PC, Cameron H, Perdue MH. Glucagon-like peptide-2 enhances intestinal epithelial barrier function of both transcellular and paracellular pathways in the mouse. Gut. 2000; 47(1):112-119.

17. Hadjiyanni I, Li KK, Drucker DJ. Glucagon-like peptide-2 reduces intestinal permeability but does not modify the onset of type 1 diabetes in the nonobese diabetic mouse. Endocrinology. 2009;150(2): 592-599.

18. Ivory CPA, Wallace LE, Mccafferty D-M, Sigalet DL. Interleukin-10independent anti-inflammatory actions of glucagon-like peptide 2. Am J Physiol Gastrointest Liver Physiol. 2008;295(6):1202-1210.

19. Guan X, Karpen HE, Stephens J, et al. GLP-2 receptor localizes to enteric neurons and endocrine cells expressing vasoactive peptides and mediates increased blood flow. Gastroenterology. 2006;130(1):150-164.

20. Yusta B, Huang L, Munroe D, et al. Enteroendocrine localization of GLP-2 receptor expression in humans and rodents. Gastroenterology 2000;119(3):744-755.

21. Ørskov C, Hartmann B, Poulsen SS, Thulesen J, Hare KJ, Holst JJ. GLP-2 stimulates colonic growth via KGF, released by subepithelial myofibroblasts with GLP-2 receptors. Regul Pept. 2005;124(1-2): $105-112$.

22. Dubé PE, Brubaker PL. Frontiers in glucagon-like peptide-2: multiple actions, multiple mediators. Am J Physiol Endocrinol Metab. 2007; 293(2):460-465.

23. Dubé PE, Forse CL, Bahrami J, Brubaker PL. The essential role of insulin-like growth factor-1 in the intestinal trophic effects of glucagonlike peptide-2 in mice. Gastroenterology. 2006;131(2):589-605.

24. Bulut K, Pennartz C, Felderbauer P, et al. Glucagon like peptide-2 induces intestinal restitution through VEGF release from subepithelial myofibroblasts. Eur J Pharmacol. 2008;578(2-3): 279-285.

25. Sigalet DL, Wallace LE, Holst JJ, et al. Enteric neural pathways mediate the anti-inflammatory actions of glucagon-like peptide 2. Am J Physiol Gastrointest Liver Physiol. 2007;293(1):211-221.
26. Rowland KJ, Brubaker PL. The 'cryptic' mechanism of action of glucagon-like peptide-2. Am J Physiol Gastrointest Liver Physiol. 2011; 301(1):G1-G8.

27. Hartmann B, Harr MB, Jeppesen PB, et al. In vivo and in vitro degradation of glucagon-like peptide-2 in humans. J Clin Endocrinol Metab. 2000;85(8):2884-2888.

28. Hartmann B, Thulesen J, Kissow H, et al. Dipeptidyl peptidase IV inhibition enhances the intestinotrophic effect of glucagon-like peptide-2 in rats and mice. Endocrinology. 2000;141(11):4013-4020.

29. Jeppesen PB, Hartmann B, Hansen BS, Thulesen J, Holst JJ, Mortensen PB. Impaired meal stimulated glucagon-like peptide 2 response in ileal resected short bowel patients with intestinal failure. Gut. 1999;45(4):559-563.

30. Jeppesen PB, Hartmann B, Thulesen J, et al. Elevated plasma glucagonlike peptide 1 and 2 concentrations in ileum resected short bowel patients with a preserved colon. Gut. 2000;47(3):370-376.

31. Jeppesen PB, Hartmann B, Thulesen J, et al. Glucagon-like peptide 2 improves nutrient absorption and nutritional status in shortbowel patients with no colon. Gastroenterology. 2001;120(4): 806-815.

32. Jeppesen PB, Sanguinetti EL, Buchman A, et al. Teduglutide (ALX0600), a dipeptidyl peptidase IV resistant glucagon-like peptide 2 analogue, improves intestinal function in short bowel syndrome patients. Gut. 2005;54(9):1224-1231.

33. Jeppesen PB, Gilroy R, Pertkiewicz M, Allard JP, Messing B, O'Keefe SJ. Randomised placebo-controlled trial of teduglutide in reducing parenteral nutrition and/or intravenous fluid requirements in patients with short bowel syndrome. Gut. 2011;60(7): 902-914.

34. Jeppesen PB, Messing B, Pertkiewicz M, Cyran J, Demchyshyn LL, Kershner R. Teduglutide, a glucagon-like peptide-2 (GLP-2) analog, improves fluid balance in short bowel syndrome (SBS) patients depending on parenteral support (PN). Gastroenterology. 2008;134(4): A110-A111.

35. Gilroy R, Allard J, Jeppesen PB, et al. Treatment out to 1 year with a GLP-2 analog, teduglutide, safely reduces parenteral nutrition (PN) needs in PN-dependent short bowel syndrome (SBS) patients. Am J Gastroenterol. 2008;103:S105-S106.

36. NPS Pharmaceuticals, Inc. A 24-week study of the efficacy and safety of teduglutide in subjects with parenteral nutrition-dependent short bowel syndrome. NPS press release. STEPS (Study of Teduglutide in PN-dependent Short-bowel syndrome). 2011. In press.

37. Henriksen DB, Alexandersen P, Bjarnason NH, et al. Role of gastrointestinal hormones in postprandial reduction of bone resorption. $J$ Bone Miner Res. 2003;18(12):2180-2189.

38. Haderslev KV, Jeppesen PB, Hartmann B, et al. Short-term administration of glucagon-like peptide-2. Effects on bone mineral density and markers of bone turnover in short-bowel patients with no colon. Scand J Gasterol. 2002;37(4):392-398.

39. Gottschalck IB, Jeppesen PB, Hartmann B, et al. Effects of treatment with glucagon-like peptide-2 on bone resorption in colectomized patients with distal ileostomy or jejunostomy and short-bowel syndrome. Scand J Gastroenterol. 2008;43(11):1304-1310.

40. Jeppesen PB, Lund P, Gottschalck IB, et al. Short bowel patients treated for two years with glucagon-like peptide-2: effects on intestinal morphology and absorption, renal function, bone and body composition, and muscle function. Gastroenterol Res Pract. 2009; 2009:616054.

41. Xiao Q, Boushey RP, Cino M, Drucker DJ, Brubaker PL. Circulating levels of glucagon-like peptide-2 in human subjects with inflammatory bowel disease. Am J Physiol Regul Integr Comp Physiol. 2000;278(4): 1057-1063.

42. Buchman AL, Katz S, Fang JC, Bernstein CN, Abou-Assi SG; for the Teduglutide Study Group. Teduglutide, a novel mucosally active analog of glucagon-like peptide-2 (GLP-2) for the treatment of moderate to severe Crohn's disease. Inflamm Bowel Dis. 2010;16(6): 962-973. 
43. Koehler JA, Yusta B, Drucker DJ. The HeLa cell glucagon-like peptide-2 receptor is coupled to regulation of apoptosis and ERK1/2 activation through divergent signaling pathways. Mol Endocrinol. 2005; 19(2):459-473.

44. Drucker DJ, Yusta B, Boushey RP, DeForest L, Brubaker PL. Human $\left[\mathrm{Gly}^{2}\right]$ GLP-2 reduces the severity of colonic injury in a murine model of experimental colitis. Am J Physiol Gastrointest Liver Physiol. 1999;276: 79-91.

45. Koehler JA, Harper W, Barnard M, Yusta B, Drucker DJ. Glucagon-like peptide-2 does not modify the growth or survival of murine or human intestinal tumor cells. Cancer Res. 2008;19(2):7897-7904
46. Rocha FG, Shen KR, Jasleen J, et al. Glucagon-like peptide-2: divergent signaling pathways. J Surg Res. 2004;121(1):5-12.

47. Iakoubov R, Lauffer LM, Trivedi S, Kim YI, Brubaker PL. Carcinogenic effects if exogenous and endogenous glucagon-like peptide- 2 in azoxymethane-treated mice. Endocrinology. 2009;150(9):4033-4043.

48. Thulesen J, Hartmann B, Hare KJ, et al. Glucagon-like peptide 2 (GLP-2) accelerates the growth of colonic neoplasms in mice. Gut. 2004;53(8):1145-1150.

\section{Publish your work in this journal}

Clinical and Experimental Gastroenterology is an international, peerreviewed, open access journal, publishing all aspects of gastroenterology in the clinic and laboratory, including: Pathology, pathophysiology of gastrointestinal disease; Investigation and treatment of gastointestinal disease; Pharmacology of drugs used in the alimentary tract;
Immunology/genetics/genomics related to gastrointestinal disease. This journal is indexed on CAS. The manuscript management system is completely online and includes a very quick and fair peer-review system. Visit http://www.dovepress.com/testimonials.php to read rea quotes from published authors.

Submit your manuscript here: http://www.dovepress.com/clinical-and-experimental-gastroenterology-journal 